\title{
Deficiencies of effectiveness of intervention studies in veterinary medicine: a cross-sectional survey of ten leading veterinary and medical journals
}

Nicola Di Girolamo, Reint Meursinge Reynders

The validity of studies that assess the effectiveness of an intervention (Eol) depends on variables such as the type of study design, the quality of their methodology, and the participants enrolled. Five leading veterinary journals and 5 leading human medical journals were hand-searched for Eol studies for the year 2013. We assessed (1) the prevalence of randomized controlled trials (RCTs) among Eol studies, (2) the type of participants enrolled, and (3) the methodological quality of the selected studies. Of 1707 eligible articles, 590 were Eol articles and 435 RCTs. Random allocation to the intervention was performed in 52\% (114/219; $95 \% \mathrm{Cl}: 45.2-58.8 \%)$ of veterinary Eol articles, against $87 \%$ (321/371; 82.5-89.7\%) of human Eol articles (adjusted OR:9.2; 3.4-24.8). Veterinary RCTs were smaller (median: 26 animals versus 465 humans) and less likely to enroll real patients, compared with human RCTs (OR:331; 45-2441). Only $2 \%$ of the veterinary RCTs, versus $77 \%$ of the human RCTs, reported power calculations, primary outcomes, random sequence generation, allocation concealment and estimation methods. Currently, internal and external validity of veterinary Eol studies is limited compared to human medical ones. To address these issues, veterinary interventional research needs to improve its methodology, increase the number of published RCTs and enroll real clinical patients. 
1 Deficiencies of effectiveness of intervention studies in

2 veterinary medicine: a cross-sectional survey of ten

3 leading veterinary and medical journals

4 Nicola Di Girolamo, DVM, MSc(Evidence-Based Health Care); ${ }^{1,2,3^{*}}$ Reint Meursinge

5 Reynders, DDS, MS, MSc(Evidence-Based Health Care) ${ }^{4,5}$

6

$7 \quad{ }^{1}$ Centro Veterinario Specialistico, Via Sandro Giovannini 53, 00137 Roma, Italy

82 Department of Veterinary Sciences, University of Bologna, Via Tolara di Sopra 50,

9 40064, Ozzano Emilia (BO), Italy

$10 \quad{ }^{3}$ EBMVet, Cremona, Italy; nicoladiggi@gmail.com

114 Private practice of orthodontics, Via Matteo Bandello 15, 20123 Milan, Italy

$12{ }^{5}$ Department of Oral and Maxillofacial Surgery, Academic Medical Center, University of

13 Amsterdam, Meibergdreef 9, 1105 AZ Amsterdam, The Netherlands;

14 reyndersmail@gmail.com

15 * Corresponding author; nicoladiggi@gmail.com; +393292003570 (ND) 


\section{ABSTRACT}

24 The validity of studies that assess the effectiveness of an intervention (Eol) depends on

25 variables such as the type of study design, the quality of their methodology, and the

26 participants enrolled. Five leading veterinary journals and 5 leading human medical

27 journals were hand-searched for Eol studies for the year 2013. We assessed (1) the

28 prevalence of randomized controlled trials (RCTs) among Eol studies, (2) the type of participants enrolled, and (3) the methodological quality of the selected studies. Of 1707 eligible articles, 590 were Eol articles and 435 RCTs. Random allocation to the intervention was performed in 52\% (114/219; 95\%Cl:45.2-58.8\%) of veterinary Eol articles, against $87 \%(321 / 371 ; 82.5-89.7 \%)$ of human Eol articles (adjusted OR:9.2; 3.4-24.8). Veterinary RCTs were smaller (median: 26 animals versus 465 humans) and less likely to enroll real patients, compared with human RCTs (OR:331; 45-2441). Only $2 \%$ of the veterinary RCTs, versus $77 \%$ of the human RCTs, reported power calculations, primary outcomes, random sequence generation, allocation concealment and estimation methods. Currently, internal and external validity of veterinary Eol studies is limited compared to human medical ones. To address these issues, veterinary interventional research needs to improve its methodology, increase the number of published RCTs and enroll real clinical patients. 


\section{Introduction}

Evaluation of medical interventions may be biased by inferential reasoning. The trustworthiness and applicability of outcomes of studies that assess the effectiveness of an intervention (EoI) strongly depend on the (1) choice of the research design, (2) the methodological quality, and (3) the characteristics of the included population (Schulz et al., 1995; Moinpour et al., 2000; Moher et al., 2010). These issues apply to both veterinary and human medicine. In this study we assessed how these three items differ between these fields.

A randomized controlled trial (RCT) is a study in which patients are randomly allocated to either an intervention or a control group (Sackett et al., 1996; Sibbald \& Roland, 1998). This design avoids most of the biases that occur in observational studies and has the potential to provide the highest quality of evidence (Moher et al., 2010). Ideally, the effectiveness of every intervention should be tested in a RCT before its implementation in clinical practice (Moher et al., 2010; Armitage, 1982). Although the importance of RCTs is universally acknowledged, the prevalence of RCTs varies between different specialties. This prevalence could be used to assess how robust is research on EoI in a specific field. For example, in periodontal research $10 \%$ of the publications between 1980 and 2000 were RCTs (Sjögren \& Halling, 2002). In nursing science, $3.8 \%$ of all articles published between 1986 and 2000 were RCTs (Lingna, 2002). In plastic surgery journals, RCTs comprised only 1.8\% of all published articles between 1990 and 2005 (Momeni et al., 2008). The prevalence of RCTs was not constant over the last 50 years (Sjögren \& Halling, 2002; Becker et al., 2008). In a survey including all specialties of internal medicine, a common ascending trend in the publication of RCTs was found between 1966 and 2001 (Strippoli et al., 2004), while between 1998 and 2002 there was no increase. Similarly, in periodontal research there was an increase in the annual number of RCTs published between 
671980 and 1994, but the number remained approximately unchanged between 1994 and 2000

68 (Sjögren \& Halling, 2002). Studies that assessed the prevalence of RCTs among EoI studies were

69

70 not identified in the published veterinary literature.

The label "randomized" is not sufficient to guarantee the methodological soundness of an EoI study. In fact, RCTs should also adhere to a wide variety of other quality parameters (Schulz et al., 1995; Moher et al., 2010). When the RCTs published in the veterinary literature were evaluated, several methodological issues were identified (Elbers \& Schukken, 1995; Lund et al., 1998; Brown, 2006; Brown, 2007; Sargeant et al., 2009; Sargeant et al., 2010; Giuffrida et al., 2012; Giuffrida, 2014). Lund et al. (1998) identified a lack of reporting of at least 2 of 6 evaluated domains (random sequence generation, informed consent, eligibility criteria, blinding, power calculation and statistical analyses) in all RCTs on dogs and cats published between 1986 and 1990. Brown et al. (1998) found that only 11\% of 97 RCTs published between 2000 and 2005 on dogs and cats reported both random sequence generation and allocation concealment. In a subset of 63 RCTs on dogs and cats, Brown et al. (2007) reported that most RCTs with losses to follow-up did not account for these losses in the data analysis and did not acknowledge the potential impact on the outcomes of these RCTs. Another study found substantive deficiencies in the reporting of key methodological domains in RCTs with dogs and cats published between 2006 and 2008 (Sargeant et al., 2010). RCTs performed in laboratory animal research showed similar methodological problems: an overview of 31 systematic reviews found that only $29 \%$ of studies reported randomization, $15 \%$ of studies reported allocation concealment, and $35 \%$ of studies reported blinded outcome assessment (Hirst et al., 2014).

Even when RCTs are conducted according to high methodological standards (i.e., strong internal validity), their applicability can be challenged by the type of patients enrolled. Outcomes 
90 of RCTs have limited external validity when the participants of the study represent only a small

91 part of the population of interest (Pressler et al., 2013), e.g., if participants are healthier than

92 patients that are not recruited (Halbert et al., 1999; Moinpour et al., 2000). This issue can affect

93 the external validity of research outcomes and limits the translation of results from RCTs to

94 "true" clinical patients.

95 The veterinary literature shares several common factors with the medical literature (e.g.,

96 presence of generalist and specialist journals). Furthermore, opinion leaders have recently

97 underscored that animal trials should be more similar to human RCTs (Muhlhausler et al., 2013).

98 We therefore decided to exploit the similarities between these two disciplines, and to use human

99 medical literature as the comparator for the veterinary medical literature.

100 The purpose of this study is to compare the veterinary literature and human medical

101 literature regarding (1) the prevalence of RCTs among EoI studies; (2) the prevalence of RCTs

102 that did not enroll clinical patients; (3) the reporting of key methodological domains in RCTs.

103 Prior to undertaking this research study we conducted scoping searches of the literature to avoid

104 research replication (Chalmers et al., 2014). These searches showed that no previous cross-

105 sectional literature reviews compared the quality of EoI articles in veterinary medicine with

106 those in human medicine.

107

108

Materials and methods

109

Design and study outcomes

110

We performed a cross-sectional analysis of the literature comparing leading veterinary

111 medical and human medical journals in the year 2013. 
113 between veterinary and human medical literature; (2) the difference in the prevalence of

114 enrollment of participants that are not real clinical patients between veterinary and human

115 medical literature; (3) the difference in reporting of key methodological domains in RCTs

116 between veterinary and human medical literature. All other outcomes are considered secondary 117 outcomes.

\section{Sample size and included journals}

We performed a pilot study to assess the prevalence of RCTs over the total number of

121

122

123

124

125

126

127

128

129

130

131

132

133

134 articles that described the effectiveness of interventions in veterinary medicine and human

medicine. All articles that were published in the first six months of 2006 in one veterinary

journal (JAVMA) and one human medical journal (JAMA) were assessed for the prevalence of

RCTs. A prevalence of 69,1\% (29 RCTs/42 intervention articles) and 29,7\% (8 RCTs/27

intervention articles) was identified for JAMA and JAVMA respectively. Using a formula for

two proportions and equal group size (Kirkewood \& Sterne, 2003) we calculated that a minimal

sample of 45 articles per group was required to have $90 \%$ power to detect a difference at a level

of statistical significance of $1 \%$. We estimated how many journals we had to search and for

which time span based on pertinent research data from a study by Giuffrida et al. (2012). Based

on the pilot study we expected to find roughly one RCT per 3 EoI articles and that each journal

should have published approximately 15 EoI articles per-year. To be conservative, we included 5

leading journals for each discipline. The "VETERINARY SCIENCES" and "MEDICINE,

GENERAL AND INTERNAL" categories of the 2013 ISI Journal Citation Report were sorted

by impact factor. All the journals that focused on sub-specialties or in non-English language

PeerJ reviewing PDF | (2015:10:7168:3:0:NEW 9 Jan 2016) 
135 were excluded. All the journals that were not published before the year 2000 or had an impact

136 factor lower than 1.0 were excluded. Aims and scopes of the remaining journals were evaluated

137 on their websites until the first 5 journals presenting broad scope were identified. Veterinary

138 journals included were: 'Veterinary Journal' (Vet J); 'Veterinary Record' (Vet Rec); 'Journal of

139 Veterinary Internal Medicine' (JVIM); 'Journal of the American Veterinary Medical

140 Association' (JAVMA); 'American Journal of Veterinary Research' (AJVR); Medical journals:

141 'New England Journal of Medicine' (NEJM); 'the Lancet'; 'Journal of the American Medical

142 Association' (JAMA); 'British Medical Journal' (BMJ); 'Annals of Internal Medicine’. Impact

143 factors ranged from 1.2 to 2.2 for veterinary medical journals and from 16.1 to 54.4 for human

144 medical journals. Details of the pilot study, sample size calculation and eligibility criteria for

145 journal inclusion are reported in Supplementary Note 1.

146

147 Data extraction published between the $1^{\text {st }}$ of January 2013 up to the $31^{\text {st }}$ of December of 2013 , including supplements, through their online archives. The total number of full original articles, EoI articles, and RCTs were recorded. EoI articles were subsequently classified based on their inclusion of real patients and type of interventions (surgical/non-surgical). RCTs were further classified based on their methodological characteristics. 
157 - Number of full original articles: primary research, including subgroup analysis or follow-ups of

158 previous articles; case series, defined as original reports including more than one patient.

159

160 - Number of articles evaluating effectiveness of interventions (EoI): "Effectiveness" was defined

161 as "evaluation of benefits" of an intervention. "Interventions" were defined as "acts used to

162 improve health, to treat a particular condition or disease in process or to prevent development of

163 a particular condition or disease" (Farlex Medical Dictionary, 2014; Merriam-Webster

164 Encyclopaedia Britannica, 2014). Studies eligible as "EoI articles" included: Case series, case-

165 control studies, cohort studies, analytical cross-sectional studies, non-randomized controlled

166 trials, and RCTs.

167

168 - Number of EoI articles that described surgical interventions: Studies of surgical interventions

169 face different challenges regarding several aspects, including study design (McCulloch, 2009).

170 To account for this factor, the type of intervention was categorized as surgical/non-surgical. EoI

171 articles were considered "surgical" when (1) the intervention required cutting of the skin.

172 Needle-related procedures (e.g., amniocentesis, etc.) were not considered surgical procedures;

173 (2) The difference between the control and the experimental group was based on the type or

174 technique of the surgical procedure. A trial was not considered "surgical" when the difference

175 between the control and the experimental group was a medication given either before or after a

176 surgical procedure.

177

178 - Number of RCTs: Studies were defined RCTs based on the US National Library of Medicine

1792008 definitions for the Publication Type terms 'Randomized Controlled Trial' and based on the 
180 definition of the Cochrane glossary (Glossary of Cochrane terms, 2014). All the reports with

181 allocation to interventions described as "randomized" were included (Schulz et al., 1994). A

182 study was classified as "a RCT" when (1) at least two interventions were compared; (2) and

183 randomization was mentioned.

- Number of RCTs that included real patients: We evaluated if RCTs involved real clinical patients or non-patients. Real clinical patients were defined as "the population that presents the condition that needs to be treated or prevented and that will benefit of the intervention once established". Non-patients refer to voluntary healthy participants or laboratory animals. Articles were considered to include real clinical patients when these individuals or animals: (1) suffered from a spontaneous disease; and (2) were exposed to real-life conditions.

The full definitions of each extracted data item are reported in Supplementary Note 2. Categorization of the EoI articles in randomized and non-randomized trials was initially performed through scrutinizing the title and the abstract of the article. If random allocation was not mentioned in the title and the abstract, the full text was searched for the term "random". Fulltexts of all the articles classified as "RCTs" were retrieved for the second phase of the study.

\section{Evaluation of the RCTs}

We assessed all the RCTs retrieved from the 5 selected veterinary journals in 2013 for the reporting of key methodological items (Chan \& Altman, 2005). Random sampling stratified by journal was performed on the medical RCTs in order to assess a number of medical RCTs in a 
20360 medical RCTs were sampled. Each medical RCT was sequentially numbered and 5 series of

20412 random numbers (one for each journal) were generated with a random number generator.

205 Two operators independently assessed the electronic full-text of each RCT and eventual

206 supporting information. In case of disagreement, an arbiter was consulted. The following key

207 methodological domains were assessed: primary outcome, power calculation, random sequence

208 generation, allocation concealment, blinding of participants, blinding of personnel, blinding of

209 outcome assessors, intention-to-treat, effect size estimation methods (Table 1). Details regarding

210 research procedures and definitions are reported in the Supplementary Note 2.

211

212 Statistical analysis

213 SPSS statistics (v22.0, IBM, Chicago, IL) was used for the statistical analysis. Statistical

214 significance was set at $\mathrm{P}<0.05$, unless otherwise specified. Prevalence of RCTs in veterinary and

215 human medical journals was calculated as: number of RCTs/total number of EoI articles.

216 Confidence intervals for proportions were estimated with the Wilson procedure with continuity

217 correction (Newcombe, 1998). Articles were the unit of the primary analysis. In the unadjusted

218 analysis the strength of the association was described as odds ratios (ORs) with their 95\%

219 confidence interval and was calculated in two-by-two tables. Chi-squared tests or Fisher tests

220 (depending on the number of expected values in each cell) were used to evaluate whether there

221 were significant differences in proportions. If one or more of the cells in the contingency table

222 were zero, a non-constant continuity correction was employed to account for the imbalance of

223 the group sizes. A factor of the reciprocal of the size of the opposite treatment group was added

224 to the cells (Sweeting et al., 2004). 

odds ratio adjusted for confounders (Peng \& So, 2002; Heck et al., 2013). In the multilevel medicine) and type of intervention (i.e., surgical/non-surgical) were included as fixed effects and journal as a random effect (Heck et al., 2013). In the logistic regression models, confounders included: type of intervention (i.e., surgical/non-surgical), type of trial (randomized/nonrandomized), type of patients enrolled (clinical patients/non-patients), and discipline (veterinary/medicine). Variables were retained in the model based on statistical significance $(\mathrm{P}<0.1)$ and based on the effect they had on the final model. To avoid overfitting of the model, a minimum of 10 events per predictor variable were required (Peduzzi et al., 1996). Goodness of fit was assessed with Hosmer-Lemshow test. Nagelkerke R squared was reported (Nagelkerke 1991). Multicollinearity was suspected with variance inflation factor $>3$ and condition index $>30$ (Midi et al., 2010). Whitney U test was used as indicated by the distribution (Lehmann, 1951). robustness and the generalizability of the association between a specific discipline and the quality of reporting of key methodological domains. Sensitivity analyses were conducted by excluding from the analysis certain types of randomized trials, in order to determine their effect on the final results. In the first sensitivity analysis, we excluded all the RCTs that did not enroll real clinical patients because an earlier study identified a lack of reporting of key methodological items in RCTS on laboratory animals (Hirst et al., 2014). In an additional sensitivity analysis we excluded all the surgical RCTs because previous research on such RCTs has found a lack of 
248 adequate reporting of key methodological items (Sinha et al., 2009). In a further analysis, we

249 included only "explicit RCTs" (i.e., randomized trials that were explicitly defined in the text as

250 "randomized controlled trial" or trials registered in a repository) because we hypothesized that

251 such RCTs would have been conducted with a special focus on high methodological standards.

252 In the final analyses, we excluded all the cross-over RCTs and cluster RCTs because these trial

253 designs have also been associated with poor reporting (Walleser et al., 2011; Straube et al.,

254 2015). Results of sensitivity analyses were reported with forest plots generated with RevMan

255 (5.3; Copenhagen: The Nordic Cochrane Centre, The Cochrane Collaboration, 2014).

256

257

Results

258

259

260

261

262

263

264

265

266

267

\section{Effectiveness of intervention articles}

A total of 1707 eligible articles were identified through hand-searching procedures in 10 selected journals for the year 2013 (Fig. 1). Of these articles, 990 were published in veterinary medical journals, and 717 in human medical journals. The distribution of eligible articles among the specific journals is depicted in Table 2 . Overall, $35 \%$ of the eligible articles $(590 / 1707)$ were EoI studies. Effectiveness of an intervention was evaluated in 22\% (219/990) of the veterinary medical articles and in 52\% (371/717) of the human medical articles.

\section{Randomized Controlled Trials}

\section{Prevalence of RCTs}


271 the various journals is reported in Table 2. EoI articles published in veterinary medical journals

272 had 5.9 times the odds of being non-randomized, compared with articles in human medical

273 journals (OR: 5.9; 95\% CI: 4 to 8.8; $\mathrm{P}<0.001$ ). In the adjusted analysis, including "type of

274 intervention" (surgical/non-surgical) as fixed effect and "journal" as random effect, the results

275 were substantially unchanged (OR: 5.6; 95\% CI: 3.1 to 9.8). Human medical journals had 6 to 15

276 times the odds of publishing a RCT compared with one of the veterinary medical journals

277 (Supplementary Table S1). When only "explicit RCTs" were considered, the prevalence of RCTs

278 declined to $21 \%$ in veterinary medicine $(47 / 219 ; 95 \% \mathrm{CI}: 16.3 \%$ to $27.6 \%)$, while the prevalence 279 remained unchanged $(87 \%)$ in human medicine.

\section{Characteristics of RCTs}

The number of subjects randomized in the articles ranged from 5 to 28244, with a median of 59 subjects (IQR: 307 subjects), a mean of 857 subjects, and a SD of 3278 subjects. Sample size was not normally distributed in veterinary and in human medical trials. Trials were significantly smaller in veterinary medical journals having a median sample size of 26 subjects (range \pm IQR: 5-28244 \pm 47 ) compared to 465.5 subjects in human medical journals (range \pm IQR: 32-27347 \pm 1267)(Mann-Whitney U: 607.0; P<0.001). Furthermore, veterinary crossover trials were significantly smaller, i.e., with a median sample size of 8 subjects (range \pm IQR: 5-20

$289 \pm 5$ ), compared with 36 subjects in other RCT designs (range \pm IQR: 6-28244 \pm 63)(Mann-

290 Whitney U: 131.5; $\mathrm{P}<0.001)$. 
Only 1 out of 114 RCTs $(0.9 \%)$ in the veterinary journals had a cluster design compared

292

293

294

295

296

297

298

299

300

301

302

303

304

305

306

307

308

309

310

311

312 Enrollment of real clinical patients

\section{Prevalence of surgical RCTs} CI: 2.6 to 6.7; $\mathrm{P}<0.001)$ (Fig. 2). 1.3 to $10.6 ; \mathrm{P}=0.01)$.

with a $13.3 \%$ in the human medical journals $(8 / 60)$. More than one fifth $(21.9 \% ; 25 / 114)$ of the veterinary RCTs had a crossover design. Of the 60 randomly sampled human medical RCTs, only one $(1.7 \% ; 1 / 60)$ had a crossover design, specifically a stepped-wedge design, i.e., cluster and crossover design. Further characteristics of RCTs are reported in the Supplementary Data.

Surgical interventions accounted for $5 \%$ of the eligible articles $(90 / 1707)$, for $15 \%$ of the EoI articles (90/590) and for $9.6 \%$ of the RCTs (42/435). Surgical interventions had 4.2 times the odds of being published as non-randomized articles than as randomized articles (OR: 4.2; 95\%

No statistically significant differences in the prevalence of surgical EoI articles were found between veterinary and human medicine (18\%; [40/219] vs 13\%; [50/371]; OR: 0.69; 95\% CI: 0.44 to $1.09 ; \mathrm{P}=0.11)$. However, veterinary RCTs had a notably lower prevalence of surgical interventions compared with veterinary EoI studies $(3.5 \% ; 4 / 114)$, while the prevalence of surgical interventions remained roughly constant among human medical EoI studies and RCTs $(11.8 \% ; 38 / 321)$. The strength of the association between surgical intervention and lack of randomization was stronger in veterinary medicine. Human medical journals had 3.7 times the odds of publishing RCTs on surgical interventions than veterinary journals (OR: $3.7 ; 95 \% \mathrm{CI}$ : 
All the human medical RCTs focused on real patients, except one (99.7\%; 320/321). Less

than a half of the veterinary RCTs $(49.1 \% ; 56 / 114)$ focused on real patients (OR: $331.4 ; 95 \%$ CI:

45 to $2441.9 ; \mathrm{P}<0.001)$.

316

The vast majority of crossover trials enrolled non-clinical patients $(84.0 \% ; 21 / 25)$, while

$41.6 \%$ of the remaining RCTs enrolled non-clinical patients (37/89). Crossover trials had 7.4

times the odds of enrolling non-clinical patients compared with other RCT designs (OR: 7.4;

95\% CI: 2.3 to $23.3 ; \mathrm{P}<0.001)$.

320

\section{Reporting of key methodological domains}

The key methodological domains were consistently reported by most human medical

RCTs, but irregularly reported by veterinary medical RCTs (Table 3). Blinding procedures of

owners, personnel and outcome assessors were the most consistently reported domains in

veterinary RCTs. None (0/114) of the veterinary RCTs adequately reported all key

methodological domains, while $23 \%(14 / 60)$ of the human medical RCTs reported all those

methodological domains (OR: 70.5; 95\% CI: 4.1 to 1208.4 ; $<<0.001)$. Only $2 \%(2 / 114)$ of the

veterinary RCTs, versus 77\% (46/60) of the human medical RCTs, adequately reported key

methodological domains that are always feasible (OR: $184 ; 95 \% \mathrm{CI}: 40.2$ to $842 ; \mathrm{P}<0.001$ ). The

number of key methodological domains adequately reported was positively correlated with the number of patients enrolled (Spearman r: 0.73; $\mathrm{P}<0.001$; Fig. 3).

A binary logistic regression model including "reporting of primary outcome", "reporting of allocation concealment", "reporting of random sequence generation", "mentioning ITT" and 
336 the source discipline (i.e., veterinary/medicine) of a RCT. "Reporting of power calculation" was

337 not included in the model because of collinearity between "reporting of the primary outcome"

338 and "reporting of the power calculation". Dependency between the variables after the removal of

339 "reporting of the power calculation" was acceptable for running the logistic regression model

340 (i.e., all condition index lower than 30.0).

341

342

343

\section{Association between clinical patients and key methodological} domains

Veterinary RCTs enrolling non-clinical patients were more likely to lack adequate reporting of each of the methodological domains evaluated in this study (Fig. 4) with the exception of blinding of personnel and intention-to-treat. The lack of an association between reporting and "intention-to-treat" should be interpreted with caution, because only 3 veterinary RCTs reported on this domain.

\section{Sensitivity and subgroup analyses}


RCTs, introduced in the early 1940s (Stuart-Harris et al., 1943; Medical Research

358 Council, 1948), have been widely adopted in clinical research to reduce the risk of subjective evaluation of effectiveness of interventions. Our study showed that the scientific rigor used to evaluate effectiveness of interventions was suboptimal in leading veterinary journals compared with general medical journals. Firstly, we observed a lower prevalence of RCTs in veterinary journals compared with medical journals (research question 1). Secondly, more than half of the RCTs in veterinary medicine did not enroll clinical patients (research question 2). Finally, key methodological domains were underreported in veterinary RCTs compared with those in medical RCTs (research question 3). This latter finding is critical as under-reporting of methodological domains in veterinary RCTs has been associated with increased treatment effects (Sargeant et al., 2010).

Observational non-randomized articles are fundamental in certain stages of the development of interventional procedures (Schünemann et al., 2013). In veterinary medicine these articles were overabundant and only about half of the articles that assessed the effectiveness of interventions was randomized. A survey in 2006 by Kuroki et al. (2009) calculated a prevalence of $34.7 \%(66 / 190)$ of RCTs on the total number of articles published in JAMA, Lancet and NEJM. In the present study we observed a prevalence of 50.6\% (260/514) of RCTs on the total number of original research articles for the same 3 journals. These data should be considered with caution, because they originated from 2 single articles that applied different sampling strategies. However, there was a $16 \%$ increase in the prevalence of RCTs in these journals between 2006 and 2013 (34.7\% vs 50.6\%; Risk difference: 0.16; 95\% CI: 0.08 to 0.24). In the present study all journals were hand-searched and all original research articles were scrutinized. Instead, Kuroki et al. (2009) obtained a random sample of the original research 
380

381

382

383

384

385

386

387

articles. Notwithstanding these differences in selection procedures, the definitions for "journal article" and "RCT" were the same in both studies. If these research studies are considered representative for their specific year of publication, the increase of the prevalence of RCTs in these medical journals in 7 years was impressive. Veterinary journals should also aim to increase the rate of published RCTs.

Experimental animal studies have been recently criticized for being inadequately conducted and reported (Hirst et al., 2014; Sena et al., 2007; Landis et al., 2012). In 2010, the REFLECT statement (Reporting guidelines for randomized controlled trials for livestock and for food safety) and the ARRIVE guidelines (Animal research: reporting of in vivo experiments) were developed for reporting RCTs of respectively livestock and laboratory animal research (Kilkenny et al., 2010; O’Connor et al., 2010). However, these guidelines apply only partly to veterinary medicine and often do not cover the needs of veterinary clinicians, i.e., they do not provide guidelines for patient-specific interventions. The finding that just one quarter of the EoI articles in the 5 veterinary journals included is a RCT on clinical patients should be a particular warning sign for veterinary clinicians. Most of the veterinary RCTs are conducted in a preclinical setting or in laboratory-controlled conditions and may not apply to the population of interest.

In the present study we observed that crossover trials were rare in leading human medical journals (1 out of 321 RCTs) but frequent in veterinary journals (more than one fifth of the RCTs). Crossover trials in veterinary medicine had a smaller sample size (median sample size of 8 patients) and were more likely to enroll non-clinical patients. The association between nonclinical patients, sample size and crossover design is straightforward in veterinary medicine: the additional expenses related to the use of experimental subjects compared with the use of clinical 
403 patients are mainly secondary to purchasing and maintaining them. Therefore, there is a tendency

404 to use the smallest "credible" sample sizes, and to apply crossover designs to provide additional

405 statistical power. The fact that only 1 out of 25 crossover trials presented a power calculation

406 supports this hypothesis. This information is not novel, as Macleod (2010) suggested "In the face

407 of pressures to reduce the number of animals used, investigators often do studies that are too

408 small to detect a significant effect."

409 In veterinary journals, parallel explicit RCTs were more likely to report power

410 calculation than crossover trials. However, only $25 \%$ of the parallel explicit RCTs reported a

411 power calculation. This is consistent with the results of a study by Giuffrida (2014), who

412 assessed trials of client-owned dogs and cats. The vast majority of medical RCTs reported power

413 calculations, which is consistent with the literature (Charles et al., 2009). Veterinary researchers

414 should apply stringent standards of statistical power when planning empirical research.

415 A statistically significant result does not provide information on the magnitude of the

416 effect and thus does not necessarily mean that the effect is robust (Landis et al., 2012). While

417 almost every RCT published in the selected human medical journals provided point estimates

418 with measures of uncertainty (i.e., 95\% CI), most articles in veterinary medicine provided just

419 these measures of statistical significance, i.e., 'P values'. Over-reliance on 'P values' when

420 reporting and interpreting results of a RCT may be inappropriate and misleading (Rothman,

421 1978; Sterne \& Davey Smith, 2001). Veterinary investigators should become more confident

422 with statistical procedures to generate point estimates and measures of uncertainty. Journal

423 editors should encourage authors to employ statistical techniques that maximize the clinical

424 interpretation of RCTs.

425 Reporting of allocation concealment in RCTs published in human medical journals 
426 improved in the last 15 years from $34.4 \%$ to $64.7 \%$ (To et al., 2013). In our study, only one tenth

427 of the RCTs published in veterinary journals mentioned allocation concealment. This is worrying

428 as there is evidence that the lack of allocation concealment is empirically associated with bias

429 (Schulz et al., 1995). Researchers should be aware of these consequences and should understand

430 that concealing treatment assignments up to the point of allocation is always possible regardless

431 of the study topic (Schulz et al., 1994). Investigators should therefore implement this issue in

432 veterinary RCTs.

433 Previous studies evaluating veterinary RCTs focused only on one journal (Elbers \&

434 Schukken, 1995), others only on some species (Lund et al., 1998; Sargeant et al., 2010) or only

435 on RCTs including client-owned animals (Brown, 2006; Brown, 2007; Giuffrida et al., 2012). To

436 maximize the external validity of this study, we included all the EoI studies. Nonetheless, the

437 present research study focused on a selected group of leading general journals. In 2006 the

438 proportion of RCTs published among JAMA, Lancet and NEJM was almost 3 times greater than

439 obstetric and gynecology journals (Kuroki et al., 2009). In addition, trials published in general

440 medical journals had higher quality scores than those in specialist journals (To et al., 2013).

441 Therefore, in this study biases are likely to overestimate the prevalence of RCTs and their

442 methodological quality compared with the remaining journals in human medicine, even if, it

443 cannot be excluded that some specialist journals have a higher prevalence of RCTs due to

444 editorial policies or other factors.

445 We found that surgical interventions were more likely investigated with non-randomized

446 trials than randomized trials in both disciplines. This is not unexpected, as surgical RCTs pose

447 more challenges than pharmaceutical RCTs (McCulloch 2009). However, the magnitude of this

448 effect may have been amplified by a selection bias. Journals included in this survey were 
449 selected for their broad scope. However, it is possible that surgical teams prefer to submit the

450 results of surgical RCTs to specialist surgical journals instead of to broad scope journals.

451 Therefore, future research should include also specialist surgical journals to provide a fairer

452 estimate of the prevalence of these randomized trials.

453 A possible weakness of this study was the non-blinded assessment of studies, i.e.,

454 reviewers knew whether they assessed a veterinary or human medical article. Even though there

455 is some evidence that blind assessments of studies might result in more consistent ratings

456 compared with open assessments (Jadad et al., 1996), some other studies suggest that blind

457 assessments provide little benefit (West, 1997; Kjaergard et al., 2001). A further weakness is the

458 limited sample of included journals. Our selection criteria led to the inclusion of the American

459 Journal of Veterinary Research (AJVR), a journal that tends to focus more on experimental than

460 clinical research. This characteristic could have led to bias toward a lower number of "real

461 patient" RCTs. However, we have performed a sensitivity analysis removing AJVR to evaluate

462 the impact of this single journal on our primary outcomes (prevalence of RCTs, prevalence of

463 real patient RCTs). We observed only a modest change in real patient RCTs (i.e., from $26 \%$ to

$46428 \%$ ), while there was a further drop in RCT prevalence in veterinary journals (i.e., from $52 \%$ to

$46545 \%$ ). Therefore, we retain that the impact of this journal on the overall results is limited and it is

466 unlikely that it has significantly biased our conclusions. Another weakness of this study is that

467 we assessed how attrition was reported and dealt with only by means of a proxy (Chan \&

468 Altman, 2005). This issue is difficult to address, because handling of attrition may be performed

469 in several different ways, and may be reported in different sections of the article (i.e., under

470 statistical analysis but also in the patient flow). As a proxy for this item, we searched for the

471 wording "intention-to-treat", based on the Cochrane glossary definition (Glossary of Cochrane 
472 terms, 2014). Obviously mentioning "intention-to-treat analysis" does not ensure that attrition 473 was properly dealt with, because the term 'intention-to-treat' could have been misused.

A limitation in the generalizability of the study is that we had to obtain a sample of

475 journals due to the number of published journals in the two disciplines $(n=133$ in the 'veterinary

476 science' category; $n=154$ in the 'medicine, general \& internal' category). This sample may not be

477 representative for the entire spectrum of journals in these fields, especially when considering 478 peculiar specialties (e.g., surgery), with exclusive issues in conduct and reporting of RCTs 479 (McCulloch, 2009). A larger sample of journals (e.g., inclusion of 10-20 journals per discipline) 480 would have been desirable to obtain results with greater generalizability.

The several limitations we have uncovered in the veterinary literature are probably secondary to the intrinsic difficulties in this field. For example, RCTs are more expensive than observational reports, and veterinary medicine has been historically allotted less funding compared with the human medical field. Furthermore, in veterinary medicine the variety of species makes performing RCTs on every species difficult. The goals in veterinary medicine are also substantially different than in human medicine, e.g. in production medicine, productivity is the primary metric of treatment success.

However, the aforementioned limitations of this study should be considered in the context of the overwhelming magnitude of the identified differences between veterinary and human medical EoI studies. Veterinary researchers should adopt medical research standards by introducing the following pathways for improvement:

(1) Education in basic evidence-based practices at all veterinary levels (i.e., undergraduates to policy-makers). Education on evidence-based practices effectively engages students (Aronoff et al., 2013). Implementing tailored courses for veterinary professionals on 
conducting, analyzing, and reporting of clinical research is an important starting point,.

496 (2) Implementation of reporting guidelines at all research levels (i.e., researchers, peer-

497

498

499

500

501

502

503

504

505

506

507

508

509

510

511

512

513

514

515

516 The objectives of this study were met, and most of the findings were alarming with regard to

517 both their number and magnitude. We observed that the veterinary literature was characterized 
518 by a lower prevalence of randomized articles compared with the human medical literature. More 519 worrying, the vast majority of the RCTs in veterinary medicine lacked adequate reporting of key 520 methodological domains (i.e., primary outcome, power calculation, random sequence generation,

521 allocation concealment, intention-to-treat) and of currently recommended statistical reporting

522 (i.e., effect measures with confidence intervals). Furthermore, RCTs published in veterinary

523 journals were less likely to enroll clinical patients than RCTs published in human medical

524 journals. These outcomes are important, because they could slow down knowledge creation and 525 could result in inappropriate claims of effectiveness of interventions. These findings should be a 526 stimulus for future researchers to improve upon the soundness of their studies. Awareness of the 527 paucity of evidence behind interventions in veterinary medicine is the first step for improvement.

Armitage, P. 1982. The role of randomization in clinical trials. Stat. Med. 1, 345-52.

Aronoff, S. C., Evans, B., Fleece, D., Lyons, P., Kaplan, L., \& Rojas, R. 2010. Integrating evidence based medicine into undergraduate medical education: combining online instruction with clinical clerkships. Teach Learn. Med. 22, 219-223. 2008. Controlled trials in aesthetic plastic surgery: a 16-year analysis. Aesthetic plastic surgery, $32(2), 359-362$.

Brown, D. C. 2006. Control of selection bias in parallel-group controlled clinical trials in dogs and cats: 97 trials (2000-2005). J. Am. Vet. Med. Assoc. 229, 990-993. 
539 Brown, D. C. 2007. Sources and handling of losses to follow-up in parallel-group randomized

540 clinical trials in dogs and cats: 63 trials (2000-2005). Am. J. Vet. Res. 68, 694-698.

541 Chalmers, I., Bracken, M.B., Djulbegovic, B., Garattini, S., Grant, J., Gülmezoglu, A.M.,

542 Howells, D.W., Ioannidis, J.P. and Oliver, S., 2014. How to increase value and reduce waste

543 when research priorities are set. The Lancet, 383(9912), pp.156-165.

544 Chan, A. W., \& Altman, D. G. 2005. Epidemiology and reporting of randomised trials published 545 in PubMed journals. Lancet. 365, 1159-1162.

546 Charles, P., Giraudeau, B., Dechartres, A., Baron, G., \& Ravaud, P. 2009. Reporting of sample

547 size calculation in randomised controlled trials: review. BMJ. 338, b1732.

548 Day, F. C., Schriger, D. L., Todd, C., \& Wears, R. L. 2002. The use of dedicated methodology

549 reviewers for peer review: a content analysis of comments to authors made by methodology and 550 regular reviewers. Ann. Emerg. Med. 40, 329-333.

551 De Angelis, C., Drazen, J.M., Frizelle, F.A., Haug, C., Hoey, J., Horton, R., Kotzin, S., Laine,

552 C., Marusic, A., Overbeke, A.J.P. and Schroeder, T.V., 2004. Clinical trial registration: a

553 statement from the International Committee of Medical Journal Editors. New England Journal of 554 Medicine, 351(12), pp.1250-1251.

555 Dickersin, K., \& Rennie, D. 2003. Registering clinical trials. JAMA. 290, 516-523.

556 Elbers, A. R., \& Schukken, Y. H. 1995. Critical features of veterinary field trials. Vet. Rec. 136, $557 \quad 187-92$.

558 Farlex Medical Dictionary. Definition: Intervention. Available: http://medical559 dictionary.thefreedictionary.com/intervention. (Accessed: June 5 2014) 
560 Giuffrida, M. A. Type II error and statistical power in reports of small animal clinical trials. $J$.

561 Am. Vet. Med. Assoc. 244, 1075-1080 (2014).

562 Giuffrida, M. A., Agnello, K. A., \& Brown, D. C. Blinding terminology used in reports of

563 randomized controlled trials involving dogs and cats. J. Am. Vet. Med. Assoc. 241, 1221-1226

564 (2012).

565 Glossary of Cochrane terms. Available: http://community.cochrane.org/glossary. (Accessed:

566 June 5 2014)

567 Halbert, J. A., Silagy, C. A., Finucane, P., Withers, R. T., \& Hamdorf, P. A. Recruitment of older

568 adults for a randomized, controlled trial of exercise advice in a general practice setting. J. Am.

569 Geriatr. Soc. 47, 477-81 (1999).

570 Heck RH, Thomas SL, Tabata LN. 2013. Multilevel Modeling of Categorical Outcomes Using

571 IBM SPSS. Routledge Academic.

572 Hirst, J.A., Howick, J., Aronson, J.K., Roberts, N., Perera, R., Koshiaris, C. and Heneghan, C., 573 2014. The need for randomization in animal trials: an overview of systematic reviews.

574 Jadad, A. R., Moore, R. A., Carroll, D., Jenkinson, C., Reynolds, D. J. M., Gavaghan, D. J., \&

575 McQuay, H. J. (1996). Assessing the quality of reports of randomized clinical trials: is blinding 576 necessary?. Controlled clinical trials, 17(1), 1-12.

577 Kilkenny, C., Browne, W. J., Cuthill, I. C., Emerson, M., \& Altman, D. G. Improving bioscience 578 research reporting: the ARRIVE guidelines for reporting animal research. PLoS Biol. 8, 579 e1000412 (2010)

580 Kirkewood, B. R., Sterne, J. A. Essential Medical Statistics. Oxford: Blackwell Science Ltd; 5812003. 
582 Kjaergard, L. L., Villumsen, J., \& Gluud, C. Reported methodologic quality and discrepancies

583 between large and small randomized trials in meta-analyses. Ann. Intern. Med. 135, 982-989

584 (2001).

585 Kuroki, L. M., Allsworth, J. E., \& Peipert, J. F. Methodology and analytic techniques used in

586 clinical research: associations with journal impact factor. Obstet. Gynecol. 114, 877-884 (2009).

587 Landis, S.C., Amara, S.G., Asadullah, K., Austin, C.P., Blumenstein, R., Bradley, E.W., Crystal, 588 R.G., Darnell, R.B., Ferrante, R.J., Fillit, H. and Finkelstein, R., 2012. A call for transparent 589 reporting to optimize the predictive value of preclinical research. Nature, 490(7419), pp.187-191.

590 Lehmann EL. Consistency and unbiasedness of certain nonparametric tests. The Annals of 591 Mathematical Statistics (1951): 165-179.).

592 Lingna, JXCYW. Literature evaluation on the randomised controlled trials and controlled 593 clinical trials published on the journal of nursing sciences. J. Nurs. Sci. 10, 002 (2002).

594 Lund, E. M., James, K. M., \& Neaton, J. D. Veterinary randomized clinical trial reporting: a 595 review of the small animal literature. J. Vet. Intern. Med. 12, 57-60 (1998).

596 Macleod, M. Why animal research needs to improve. Nature. 477, 511 (2011).

597 McCulloch, P. No surgical innovation without evaluation: the IDEAL recommendations. Lancet.

598 374, 1105-1112. (2009).

599 Medical Research Council. Streptomycin treatment of pulmonary tuberculosis: a Medical

600 Research Council investigation. BMJ. 2, 769-782 (1948).

601 Merriam-Webster Encyclopaedia Britannica. Definition: Intervention. Available:

602 http://www.merriam-webster.com/medical/intervention. (Accessed: June 5 2014) 
603 Midi, H., Sarkar, S. K., \& Rana, S. 2010. Collinearity diagnostics of binary logistic regression 604 model. Journal of Interdisciplinary Mathematics, 13(3), 253-267.).

605 Moher, D., Hopewell, S., Schulz, K.F., Montori, V., Gøtzsche, P.C., Devereaux, P.J., Elbourne, 606 D., Egger, M. and Altman, D.G., 2010. CONSORT 2010 explanation and elaboration: updated 607 guidelines for reporting parallel group randomised trials. Journal of clinical epidemiology, 63(8), 608 pp.e1-e37.

609 Moinpour, C.M., Lovato, L.C., Thompson, I.M., Ware, J.E., Ganz, P.A., Patrick, D.L., 610 Shumaker, S.A., Donaldson, G.W., Ryan, A. and Coltman, C.A., 2000. Profile of men 611 randomized to the prostate cancer prevention trial: baseline health-related quality of life, urinary 612 and sexual functioning, and health behaviors. Journal of clinical oncology, 18(9), pp.1942-1953.

613 Momeni, A., Becker, A., Antes, G., Diener, M. K., Blümle, A., \& Stark, G. B. Evidence-based 614 plastic surgery: controlled trials in three plastic surgical journals (1990-2005). Ann. Plast. Surg. 615 61, 221-225 (2008).

616 Muhlhausler, B. S., Bloomfield, F. H., \& Gillman, M. W. Whole animal experiments should be 617 more like human randomized controlled trials. PLoS Biol. 11, e1001481 (2013).

618 Nagelkerke, N. J. (1991). A note on a general definition of the coefficient of determination.

619 Biometrika, 78(3), 691-692.

620 Newcombe, R. G. Two-sided confidence intervals for the single proportion: comparison of seven 621 methods. Stat. Med. 17, 857-872 (1998).

622 O’Connor, A.M., Sargeant, J.M., Gardner, I.A., Dickson, J.S., Torrence, M.E., Dewey, C.E., 623 Dohoo, I.R., Evans, R.B., Gray, J.T., Greiner, M. and Keefe, G., 2010. The REFLECT 624 Statement: Methods and Processes of Creating Reporting Guidelines for Randomized Controlled 
625 Trials for Livestock and Food Safety by Modifying the CONSORT Statement $\uparrow$. Zoonoses and 626 public health, 57(2), pp.95-104.

627 Peduzzi, P., Concato, J., Kemper, E., Holford, T. R., \& Feinstein, A. R. A simulation study of the 628 number of events per variable in logistic regression analysis. J. Clin. Epidemiol. 49, 1373-1379 629 (1996).

630 Peng, C. Y. J., \& So, T. S. H. Logistic regression analysis and reporting: A primer. Underst. Stat. $631 \quad 1,31-70(2002)$.

632 Pressler, T. R., \& Kaizar, E. E. The use of propensity scores and observational data to estimate 633 randomized controlled trial generalizability bias. Stat. Med. 32, 3552-3568 (2013).

634 Rothman, K. J. A show of confidence. N. Engl. J. Med. 299, 1362-1363. (1978).

635 Sackett, D. L., Rosenberg, W. M., Gray, J. A., Haynes, R. B., \& Richardson, W. S. Evidence 636 based medicine: what it is and what it isn't. BMJ. 312, 71-72 (1996).

637 Sargeant, J.M., Elgie, R., Valcour, J., Saint-Onge, J., Thompson, A., Marcynuk, P. and Snedeker, 638 K., 2009. Methodological quality and completeness of reporting in clinical trials conducted in 639 livestock species. Preventive veterinary medicine, 91(2), pp.107-115.

640 Sargeant, J.M., Thompson, A., Valcour, J., Elgie, R., Saint-Onge, J., Marcynuk, P. and Snedeker, 641 K., 2010. Quality of reporting of clinical trials of dogs and cats and associations with treatment 642 effects. Journal of veterinary internal medicine, 24(1), pp.44-50.

643 Schulz, K. F., Chalmers, I., Grimes, D. A., \& Altman, D. G. Assessing the quality of 644 randomization from reports of controlled trials published in obstetrics and gynecology journals. $645 J A M A .272,125-8$ (1994). 
646 Schulz, K. F., Chalmers, I., Hayes, R. J., \& Altman, D. G. Empirical evidence of bias.

647 Dimensions of methodological quality associated with estimates of treatment effects in 648 controlled trials. JAMA. 273, 408-412 (1995).

649 Schünemann, H.J., Tugwell, P., Reeves, B.C., Akl, E.A., Santesso, N., Spencer, F.A., Shea, B., 650 Wells, G. and Helfand, M., 2013. Non-randomized studies as a source of complementary, 651 sequential or replacement evidence for randomized controlled trials in systematic reviews on the 652 effects of interventions. Research Synthesis Methods, 4(1), pp.49-62.

653 Sena, E., van der Worp, H. B., Howells, D., \& Macleod, M. How can we improve the pre654 clinical development of drugs for stroke? Trends Neurosci. 30, 433-439 (2007).

655 Sibbald, B., \& Roland, M. Understanding controlled trials. Why are randomised controlled trials 656 important? BMJ. 316, 201 (1998).

657 Sinha S, Sinha S, Ashby E, Jayaram R, Grocott MP. Quality of reporting in randomized trials 658 published in high-quality surgical journals. J Am Coll Surg. 2009 Nov;209(5):565-571.e1.

659 Sjögren, P., \& Halling, A. Randomised controlled trials and publication trends in periodontal 660 research during 1980-2000. J. Clin. Periodontol. 29, 1112-7 (2002).

661 Sterne, J. A., \& Davey Smith, G. Sifting the evidence-what's wrong with significance tests? 662 BMJ. 322, 226-231 (2001).

663 Straube S, Werny B, Friede T. A systematic review identifies shortcomings in the reporting of 664 crossover trials in chronic painful conditions. J Clin Epidemiol. 2015 Dec;68(12):1496-503.

665 Strippoli, G. F., Craig, J. C., \& Schena, F. P. The number, quality, and coverage of randomized 666 controlled trials in nephrology. J. Am. Soc. Nephrol. 15, 411-419 (2004). 
667 Stuart-Harris, C. H., Francis, A. E., \& Stansfeld, J. M. Patulin in the common cold. Lancet. 242, $668684(1943)$.

669 Sweeting, M. J., Sutton, A. J., \& Lambert, P. C. What to add to nothing? Use and avoidance of 670 continuity corrections in meta-analysis of sparse data. Stat. Med. 23, 1351-1375 (2004).

671 To, M. J., Jones, J., Emara, M., \& Jadad, A. R. Are reports of randomized controlled trials 672 improving over time? A systematic review of 284 articles published in high-impact general and 673 specialized medical journals. PLoS One. 8, e84779 (2013).

674 Walleser S, Hill SR, Bero LA. Characteristics and quality of reporting of cluster randomized 675 trials in children: reporting needs improvement. J Clin Epidemiol. 2011 Dec;64(12):1331-40. 676 doi: 10.1016/j.jclinepi.2011.04.006. Epub 2011 Jul 20.

677 West, R. Does blinding of readers affect results of meta-analyses? Lancet. 350, 892 (1997).

678

\section{Acknowledgments}

680

681

682

683

684

685

686

The authors acknowledge Paolo Selleri for his critical review and comments and Lucy Nicole Papa Caminiti for assistance with data extraction.

\section{Author contributions statement}

N.D. conceived the study, N.D. and R.M.R. conducted the study, N.D. analyzed the results. All authors reviewed the manuscript. 


\section{Additional information}

688 This manuscript represents a portion of a thesis submitted by Dr. Di Girolamo to University of

689 Oxford in partial fulfillment of the requirement for the Master of Science in Evidence Based

690 Health Care degree. The authors disclose any financial interests with companies that

691 manufacture products that are the subject of the present research or with companies that

692 manufacture competing products. 


\section{Figures}

694

695 Figure 1. Study flow.

696

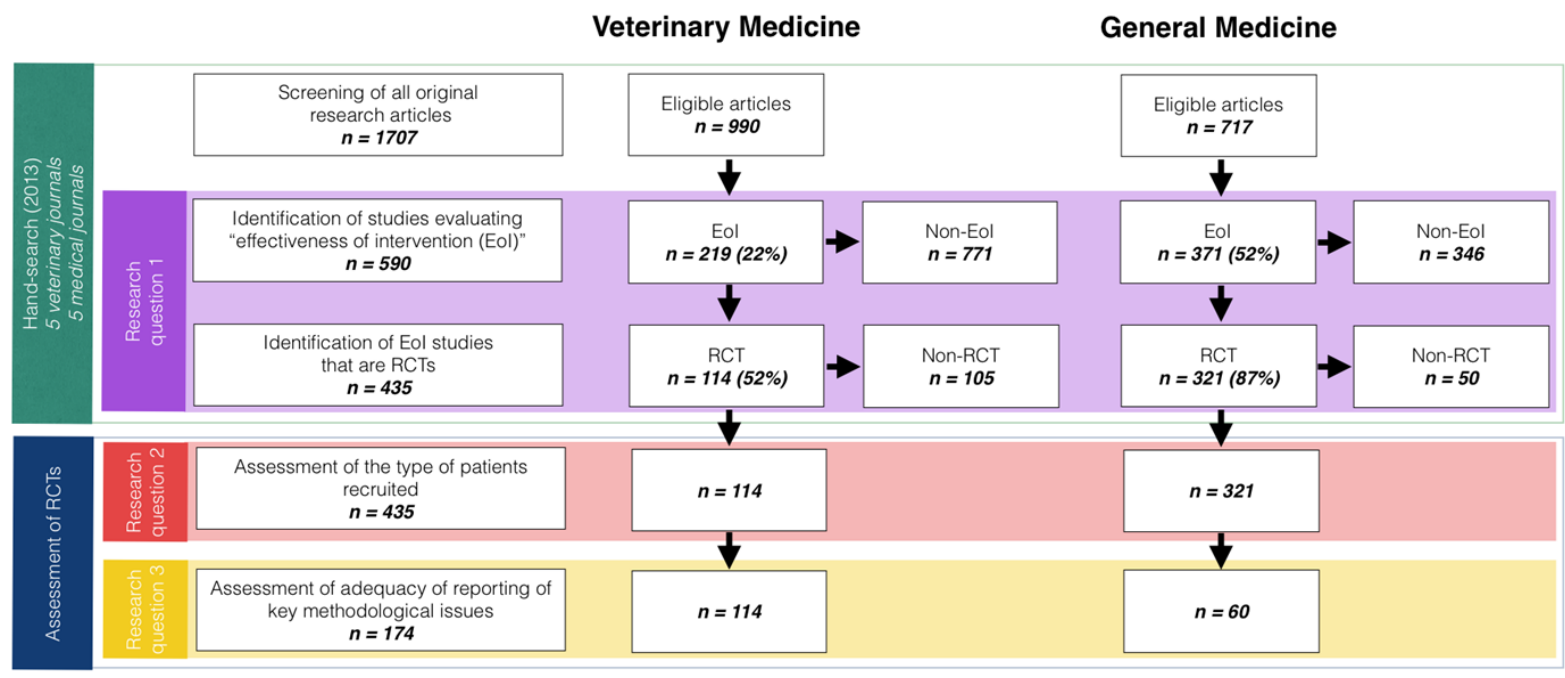

698

699 
701 Figure 2. Association (odds ratio and 95\% confidence intervals) between

702 randomization, discipline and type of intervention (surgical/non-surgical). Notice that the

703 overall prevalence of randomized controlled trials (RCTs) and prevalence of surgical

704 RCTs were lower in veterinary medicine (ORs: 4.2 and 3.7, respectively). However, 705 surgical interventions were more likely to be non-randomized in both disciplines (ORs:

$706 \quad 14.3$ and 2.3).

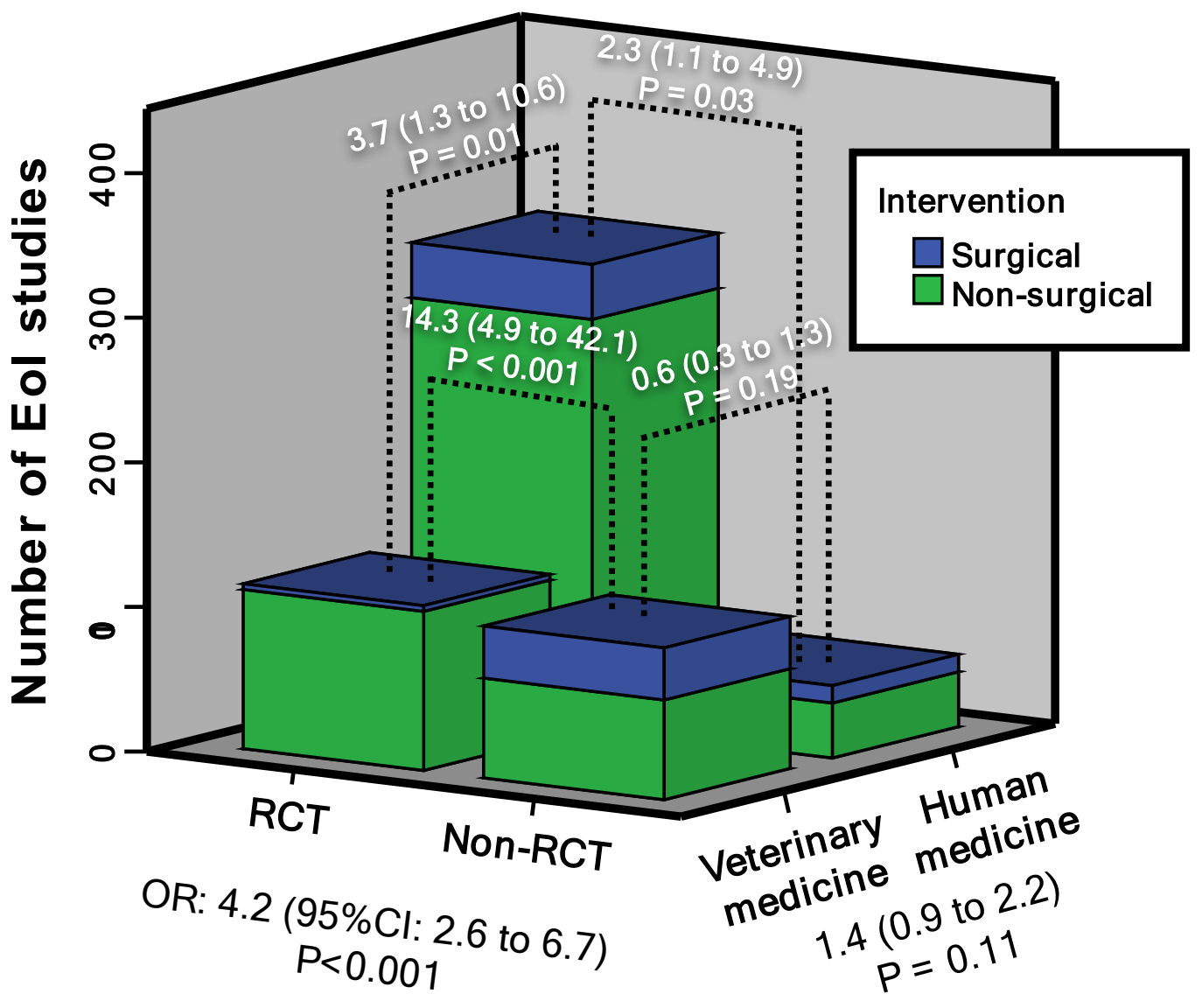


711 Figure 3. Number of patients randomized in RCTs and methodological quality. A712 Difference in number of patients randomized in veterinary and general medicine. B-

713 Difference in number of patients randomized in cross-over and other study designs. C714 Correlation between the number of methodological issues (primary outcome, power 715 calculation, random sequence generation, allocation concealment, estimation methods, 716 intention-to-treat) reported and number of patients enrolled in each RCT. Notice that the $717 \mathrm{y}$-axis were plotted on a logarithmic function.
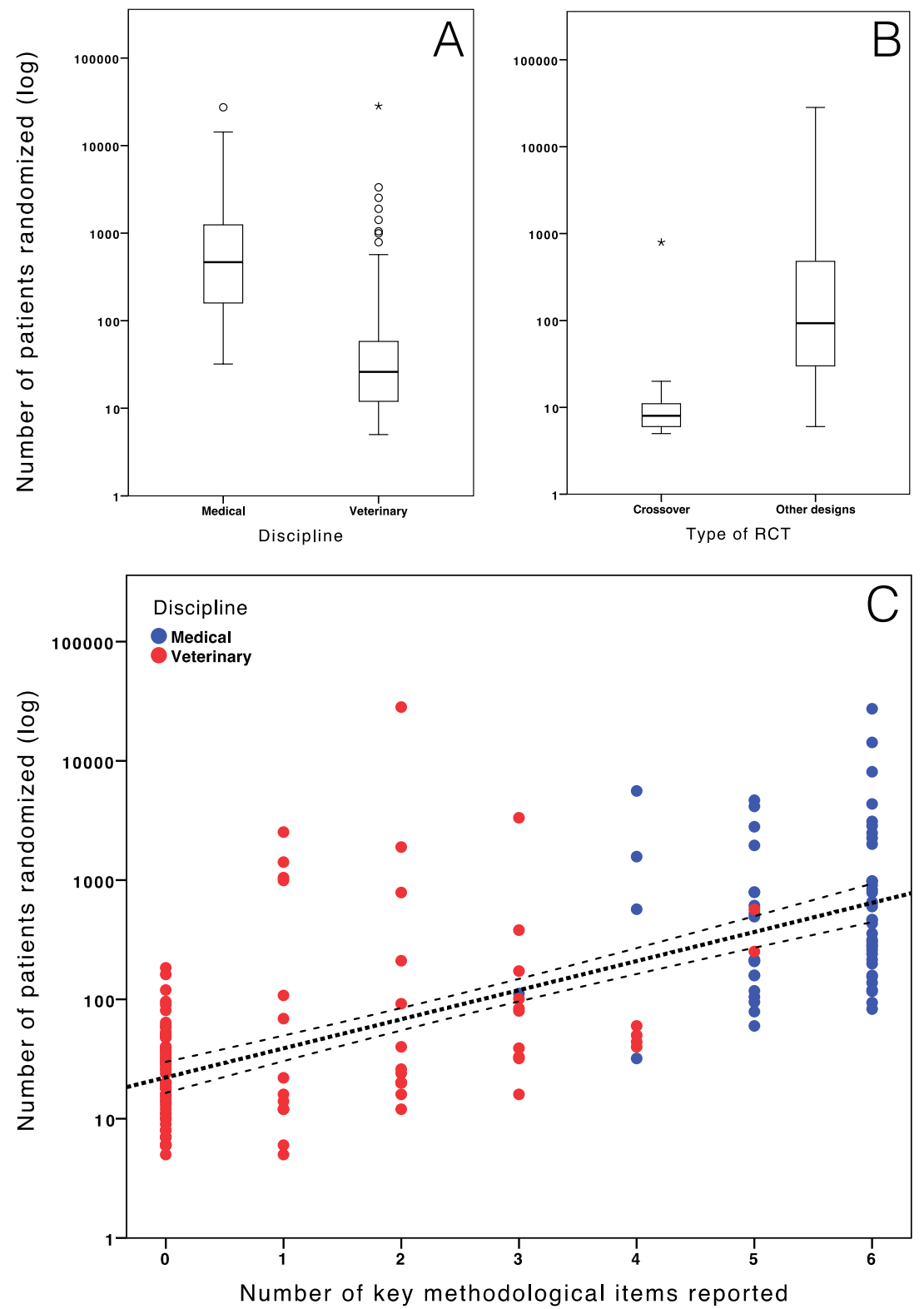
721 Figure 4. Association between type of patients enrolled (real clinical patients vs non722 patients) and adequacy of reporting of key methodological issues in RCTs published in 723 leading veterinary journals $(n=114)$. M-H: Mantel-Haenszel; $\mathrm{Cl}$ : Confidence interval.

724

\section{Odds Ratio}

Study or Subgroup

Power calculations

Explicit primary outcome

Proper random sequence

Allocation concealed

Blinding owners/patients

Blinding personnel

Blinding assessors

Intention-to-treat

Estimation methods
M-H, Fixed, 95\% Cl

12.21 [2.67, 55.87]

$9.41[2.60,34.10]$

3.78 [1.36, 10.47]

13.93 [1.73, 111.99]

$9.33[2.01,43.30]$

$1.92[0.84,4.39]$

$2.35[1.11,4.98]$

$2.11[0.19,23.96]$

24.85 [3.17, 194.46]

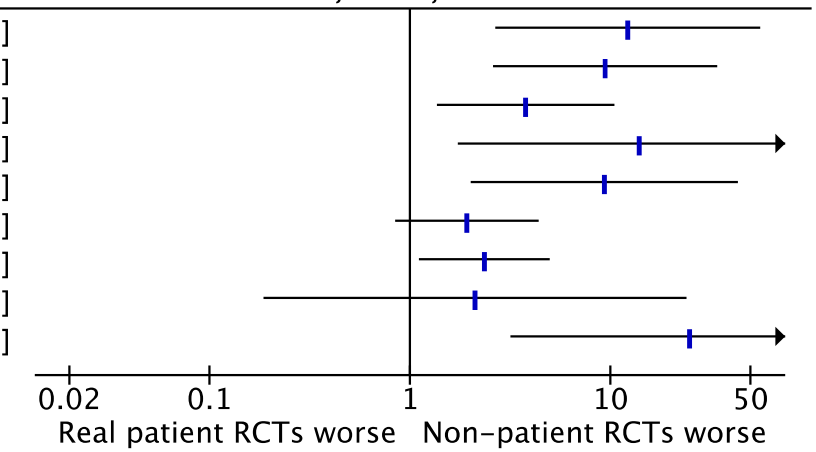

Odds Ratio

M-H, Fixed, 95\% CI

726

727

728

729 
730 Figure 5. Sensitivity and subgroup analysis of the association between discipline and 731 reporting of key methodological items. Primary analysis. Inclusion of explicit RCTs. 732 Exclusion of surgical RCTs. Inclusion of parallel explicit RCTs. Exclusion of non-patient 733 RCTs. M-H: Mantel-Haenszel; Cl: Confidence interval.

\begin{tabular}{|c|c|c|c|c|c|}
\hline Study or Subgroup & $\begin{array}{l}\text { Odds Ratio } \\
\text { M-H, Fixed, } 95 \% \mathrm{Cl}\end{array}$ & & & $\begin{array}{l}\text { Is Ratio } \\
\text { xed, } 95 \% \text { Cl }\end{array}$ & \\
\hline Primary analysis & & & & & \\
\hline $\begin{array}{l}\text { Power calculations } \\
\text { Explicit primary outcome } \\
\text { Proper random sequence } \\
\text { Allocation concealed } \\
\text { Blinding owners/patients } \\
\text { Blinding personnel } \\
\text { Blinding assessors } \\
\text { Intention-to-treat } \\
\text { Estimation methods }\end{array}$ & $\begin{array}{r}295.00[38.48,2261.83] \\
246.73[32.39,1879.54] \\
55.39[18.20,168.54] \\
42.50[17.20,105.04] \\
6.13[2.95,12.73] \\
1.32[0.68,2.58] \\
2.75[1.39,5.43] \\
164.82[44.02,617.06] \\
101.33[28.59,359.19]\end{array}$ & & & $t_{+}$ & $\stackrel{+}{\longrightarrow+}$ \\
\hline Only real patient RCTs & & 0.001 & 0.1 & $1 \quad 10$ & 1000 \\
\hline $\begin{array}{l}\text { Power calculations } \\
\text { Explicit primary outcome } \\
\text { Proper random sequence } \\
\text { Allocation concealed } \\
\text { Blinding owners/patients } \\
\text { Blinding personnel } \\
\text { Blinding assessors } \\
\text { Intention-to-treat } \\
\text { Estimation methods }\end{array}$ & $\begin{array}{r}135.35[17.30,1058.74] \\
114.89[14.75,894.69] \\
32.12[10.03,102.80] \\
20.45[7.94,52.70] \\
3.00[1.36,6.60] \\
0.97[0.45,2.08] \\
1.78[0.81,3.90] \\
120.27[25.39,569.77] \\
43.59[11.96,158.85]\end{array}$ & & & ${ }_{+1}^{+1}$ & $\stackrel{\square}{\longrightarrow+}$ \\
\hline Only non-surgical RCTs & & 0.001 & 0.1 & $1 \quad 10$ & 1000 \\
\hline $\begin{array}{l}\text { Power calculations } \\
\text { Explicit primary outcome } \\
\text { Proper random sequence } \\
\text { Allocation concealed } \\
\text { Blinding owners/patients } \\
\text { Blinding personnel } \\
\text { Blinding assessors } \\
\text { Intention-to-treat } \\
\text { Estimation methods }\end{array}$ & $\begin{array}{r}255.56[33.13,1971.09] \\
225.00[29.32,1726.76] \\
64.00[18.22,224.84] \\
33.48[13.41,83.60] \\
7.71[3.55,16.74] \\
1.63[0.82,3.26] \\
2.31[1.14,4.70] \\
221.40[46.52,1053.78] \\
134.03[29.74,603.96]\end{array}$ & & & $\underbrace{1}_{1}$ & $\begin{array}{r}\vec{\longrightarrow} \\
\stackrel{+}{\longrightarrow} \\
+\end{array}$ \\
\hline Only explicit RCTs & & 0.001 & 0.1 & $1 \quad 10$ & 1000 \\
\hline $\begin{array}{l}\text { Power calculations } \\
\text { Explicit primary outcome } \\
\text { Proper random sequence } \\
\text { Allocation concealed } \\
\text { Blinding owners/patients } \\
\text { Blinding personnel } \\
\text { Blinding assessors } \\
\text { Intention-to-treat } \\
\text { Estimation methods }\end{array}$ & $\begin{array}{r}193.09[23.91,1559.03] \\
125.87[15.89,996.94] \\
20.63[6.40,66.46] \\
18.50[6.98,49.00] \\
2.36[1.05,5.27] \\
0.67[0.30,1.46] \\
1.29[0.56,2.98] \\
100.23[21.06,476.98] \\
49.69[13.20,187.00]\end{array}$ & & & 5 & $\begin{array}{r}\longrightarrow \\
\stackrel{\longrightarrow}{\longrightarrow} \\
\stackrel{\longrightarrow}{\longrightarrow}\end{array}$ \\
\hline Only parallel explicit RCTs & & 0.01 & 0.1 & 1 & 100 \\
\hline $\begin{array}{l}\text { Power calculations } \\
\text { Explicit primary outcome } \\
\text { Proper random sequence } \\
\text { Allocation concealed } \\
\text { Blinding owners/patients } \\
\text { Blinding personnel } \\
\text { Blinding assessors } \\
\text { Intention-to-treat } \\
\text { Estimation methods }\end{array}$ & $\begin{array}{r}150.00[18.28,1230.91] \\
92.86[11.56,745.78] \\
75.00[9.39,599.19] \\
48.00[12.22,188.61] \\
3.21[1.32,7.79] \\
1.08[0.46,2.52] \\
1.96[0.77,4.97] \\
88.67[18.01,436.44] \\
57.17[11.93,274.04]\end{array}$ & & & $\square_{1}^{1}$ & $\underset{ }{\stackrel{\longrightarrow}{\longrightarrow}}$ \\
\hline & & $\begin{array}{l}0.01 \\
\text { Ger }\end{array}$ & $\begin{array}{l}0.1 \\
\text { al mec }\end{array}$ & 1 Veterinary & $\begin{array}{l}100 \\
\text { licine }\end{array}$ \\
\hline
\end{tabular}




\section{Supplementary Information}

Supplementary Note 1. Details on pilot study, sample size calculation and eligibility criteria for

743 Supplementary Note 2. Additional details on data extraction.

744 Supplementary Data. Other characteristics of RCTs evaluated.

Supplementary Table S1. Binary logistic regression outcome.

\section{Tables}

Table 1. Definitions used to assess characteristics of publications from 5 leading veterinary and 5 leading medical journals in 2013. Reporting of methodological domains was assessed in all the randomized controlled trials (RCTs) extracted. Full definition of each item is given in the main text and supplementary files. EoI: Effectiveness of intervention.

\section{Terms}

Characteristics of Original articles articles

\section{Descriptions}

Primary research, including subgroup analyses, followups of previous article and case series

Effectiveness of intervention (EoI)

articles

Randomized controlled trials (RCT) Real patients RCTS

Primary research evaluating the benefits of an intervention

EoI studies with allocation to interventions reported as randomized

RCTs that included individuals or animals that suffered from a spontaneous disease and were exposed to real-life conditions

Surgical (RCT/EoI) articles Same as previous definitions, but evaluating the benefits Explicit $R C T$ of a surgical intervention Trials registered in a trial repository or self-defining "randomized controlled trial"

Explicit parallel Same as pervious, but employing only two arms

$R C T$

Standalone RCT Lack of additional non-randomized work (i.e., in vitro or prospective data) reported in the same article of the RCT Crossover RCT $\mathrm{RCT}$ in which participants receive a sequence of different 
Cluster RCT

Key methodological domains evaluated Power calculation in RCTs
Random sequence generation

Allocation concealment

Blinding of participants Blinding of personnel

Blinding of outcome assessors Intention-to-treat

Effect size estimation methods treatments

$\mathrm{RCT}$ in which groups of participants are randomized to different treatments

A primary outcome is explicitly reported in the published article

A power calculation performed a priori to estimate the sample size is explicitly reported

Methods employed to generate the random list and type of randomization are explicitly reported

Methods used to prevent the individuals enrolling trial participants from knowing or predicting the allocation sequence in advance are explicitly described in the article

Explicit description that participants/pet owners were unaware of participants' group allocation

Explicit description that operators involved in the care of participants were unaware of participants' group of allocation

Explicit description that outcome assessors were unaware of participants' group of allocation

Explicit mention that the analysis was made on an "intention-to-treat" basis.

Results are reported with methods that estimate the effect size with confidence interval. 
759 Table 2. Number of eligible articles, EoI articles and prevalence of RCTs in 10 leading veterinary and 760 medical journals in 2013. AJVR: American Journal of Veterinary Research; JAVMA; Journal of the

761 American Veterinary Medical Association; JVIM: Journal of Veterinary Internal Medicine; Vet J:

762 Veterinary Journal; Vet Rec: Veterinary Record; Annals: Annals of Internal Medicine; BMJ: British

763 Medical Journal; JAMA: Journal of the American Medical Association; NEJM: New England Journalof

764 Medicine.

765

\begin{tabular}{|c|c|c|c|c|c|c|c|c|c|c|c|c|}
\hline & \multicolumn{6}{|c|}{ Veterinary Journals } & \multicolumn{6}{|c|}{ Medical Journals } \\
\hline & \multicolumn{3}{|c|}{ JAVM } & \multirow[b]{2}{*}{ Vet J } & \multirow{2}{*}{ Vet Rec } & \multirow{2}{*}{ Total } & \multirow[b]{2}{*}{ Annals } & \multirow[b]{2}{*}{ BMJ } & \multirow[b]{2}{*}{ JАMA } & \multirow[b]{2}{*}{ Lancet } & \multirow[b]{2}{*}{ NEJM } & \multirow[b]{2}{*}{ Total } \\
\hline & AJVR & $\mathbf{A}$ & JVIM & & & & & & & & & \\
\hline $\begin{array}{l}\text { Eligible } \\
\text { articles }\end{array}$ & 193 & 188 & 156 & 320 & 133 & 990 & 75 & 128 & 157 & 152 & 205 & 717 \\
\hline EoI articles & 43 & 51 & 40 & 59 & 26 & 219 & 31 & 46 & 71 & 94 & 129 & 371 \\
\hline RCT (n) & 34 & 18 & 19 & 30 & 13 & 114 & 24 & 37 & 61 & 84 & 115 & 321 \\
\hline$(\%)$ & $79 \%$ & $35 \%$ & $47 \%$ & $51 \%$ & $50 \%$ & $52 \%$ & $77 \%$ & $80 \%$ & $86 \%$ & $89 \%$ & $89 \%$ & $87 \%$ \\
\hline Non-RCT (n) & 9 & 33 & 21 & 29 & 13 & 105 & 7 & 9 & 10 & 10 & 14 & 50 \\
\hline$(\%)$ & $21 \%$ & $65 \%$ & $53 \%$ & $49 \%$ & $50 \%$ & $48 \%$ & $23 \%$ & $20 \%$ & $14 \%$ & $11 \%$ & $11 \%$ & $13 \%$ \\
\hline 766 & & & & & & & & & & & & \\
\hline 767 & & & & & & & & & & & & \\
\hline 768 & & & & & & & & & & & & \\
\hline 769 & & & & & & & & & & & & \\
\hline
\end{tabular}


770 Table 3. Reporting of key methodological items in RCTs published in leading veterinary and human 771 medical journals. OR: Odds ratio; CI: Confidence interval.

772

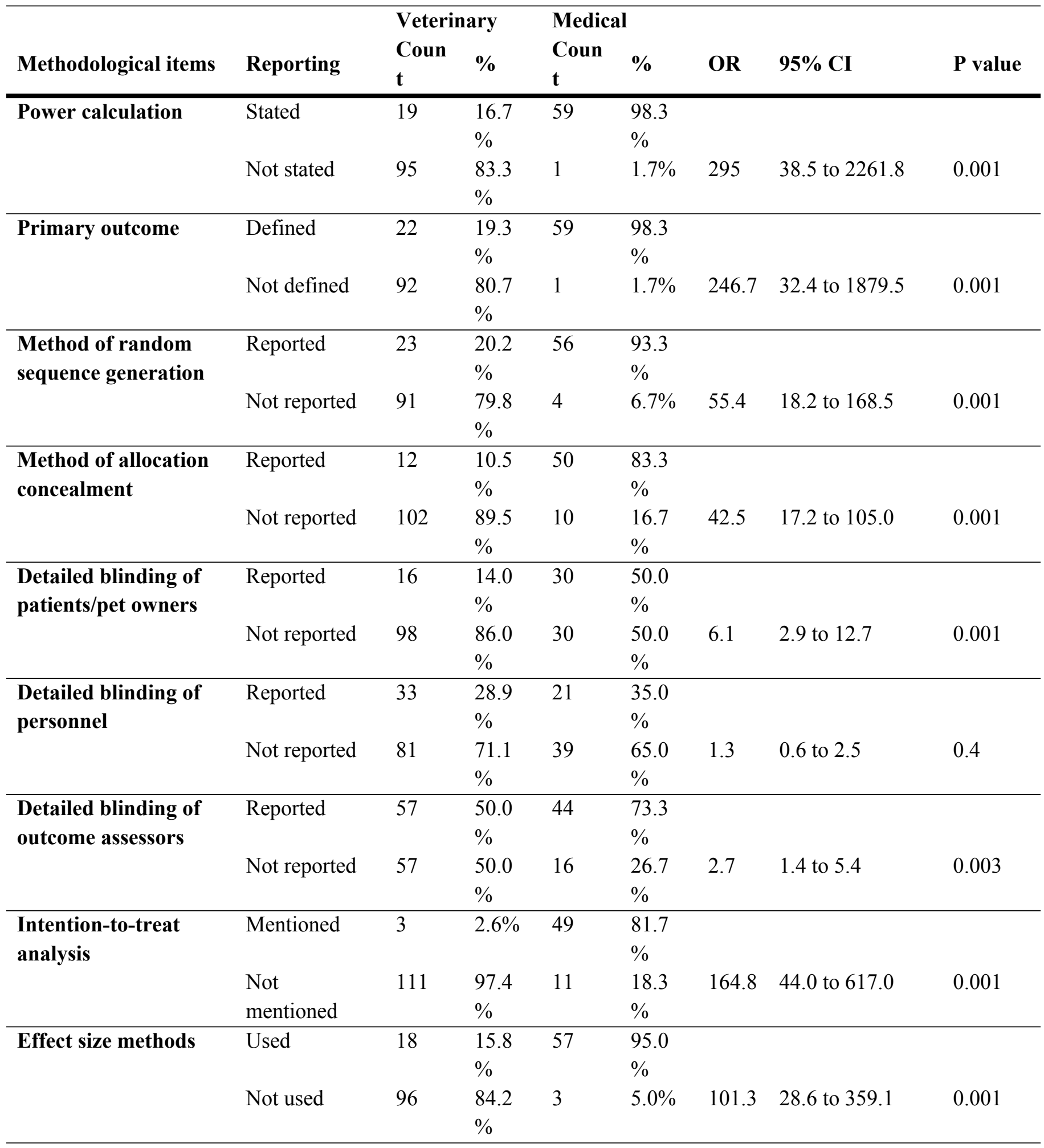

